\title{
Effect of Height on Peoples Body Specific Representation and Association between Valence and Space
}

\author{
Xianqing Liu1, Ruoqian Zhu'², Ningtianyu Wang ${ }^{3}$ \\ ${ }^{1}$ Wuhan University, Wuhan, China \\ ${ }^{2}$ Keystone Academy, Beijing, China \\ ${ }^{3}$ Southlands Schools, Qingdao, China \\ Email: ds2018@foxmail.com
}

How to cite this paper: Liu, X. Q., Zhu, R. Q., \& Wang, N. T. Y. (2019). Effect of Height on Peoples Body Specific Representation and Association between Valence and Space. Psychology, 10, 1353-1360. https://doi.org/10.4236/psych.2019.1010086

Received: April 25, 2019

Accepted: July 23, 2019

Published: July 26, 2019

Copyright $\odot 2019$ by author(s) and Scientific Research Publishing Inc. This work is licensed under the Creative Commons Attribution International License (CC BY 4.0).

http://creativecommons.org/licenses/by/4.0/ (c) () Open Access

\begin{abstract}
The body-specificity hypothesis proposes that people with different kinds of bodies tend to think differently in predictable ways (Casasanto, 2009). Previous researches have shown that people tend to make a good judgment on things on the side of their dominant hand which experiences greater motor fluency, and bad judgment on their nondominant side. This conceptual mapping has been shown to be flexible: changing the relative fluency of the hands, or even observing a change in someone else's motor fluency, results in a reversal of the mapping, such that good things become associated with the side of the nondominant hand (Casasanto \& Chrysikou, 2011). The present study consistently tests the body-specificity hypothesis in using people's height as a test bed. In Casasanto (2009) study, it is shown that about $90 \%$ of participants relate "up" with "good". Is there any different pattern of the association between space and valence among tall and short people? In the present study, we assume that taller people are more likely to make eyes look down and shorter people are more likely to look upward, and the "up is good" effect has interaction with people's height, which means heights moderate the overall association of good with up. The pre-test is to confirm the premise that taller people feel more comfortable looking down and shorter people feel more comfortable looking up. The experiments are testing whether the association of good with up is weaker in tall people and stronger in tall people. The significant effect is expected to support the body-specificity theory.
\end{abstract}

\section{Keywords}

Body-Specificity Theory, Height, Valance Judgement, Human Cognition 


\section{Introduction}

In our daily life we could easily find some clue that "up" is usually associated with "good": the top rank is always written above the bottom rank, we always use some up orientational words to express something good like "cheer up" "look up to", and some cultural evidence is found to support this mental metaphor (Lakoff \& Johnson, 1980). However, things may be much complicated when considering different body characteristics among individuals, since there is possibility that people with different height may regard up and down differently according to body-specificity hypothesis which claims that people with different body features think differently. It is feasible that less tall people related "up" with good things or feelings than short people, because of the assumption that tall people are less familiar with, or less frequently to see the upper visual field. In order to investigate this complexity, the present study goes further on the effect of height on the relationship between the vertical space and the valence judgment.

According to the body-specificity hypothesis, it has long been proposed that people who consist of different bodily characteristics should correspondingly reflect mental representations in systematically different ways (Casasanto, 2009). This has been concluded based on the premise of embodiment theory that thinking is proceeded by simulating bodily states (Barsalou, et al. 2003). Taking handedness as an example, right-handers activate their left-hemisphere motor areas when they perform actions of their dominant hand, while left-handers activate their right-hemisphere instead. Furthermore, previous researches have tested that the same portion in people's motor system would also be stimulated even if they only imagine the action instead of actually perform it. This was found by using functional magnetic resonance imaging (fMRI) to supervise people's brain motor system activities when they were imagining an action while lying perfectly still in the fMRI scanner (Casasanto \& Chrysikou, 2011). Based on this finding, other existing studies have also proposed and demonstrated some patterns of people's diverse perceptions according to their different physical characteristics. For instance, although in most of the conventions, people tend to regard the right side as good while the left side as bad, Casasanto and Chrysikou (2011) prove that people would actually favor things that are relatively more approachable or familiar to them. In that study, right-handers, whose motor system is more fluent when they interact with right sides associate "good" with "right", whereas left-handers associate "good" with "left" because they have the motor fluency when they are using their dominant hand (Casasanto \& Chrysikou, 2011). Another relevant study changes participants' motor-fluency temporarily and observes the same pattern. They asked some right-handers to wear a cumbersome glove on their right hands to make them become left-handers for 12 minutes, while the rest of the participants wear it on their left hands which maintain their innate motor-fluency. Later, all participants removed their gloves and took a space-valence association test which reveals that people who wore a 
glove on their left hand remained the preference of "right". In contrast, people who wore the right gloves think "left" is "good" like natural lefties. This result suggests that it is quite flexible for the adjustments of people's general judgments since a brief experience could vary the implicit association between space and emotional valence in terms of a reversal of their usual arbitration (Casasanto \& Chrysikou, 2011).

The present study will be consistently testing the body-specificity hypothesis but use people's height as a test bed. Casasanto (2009) has shown that $90 \%$ of people relate "up" with "good". However, the previous studies mentioned give us inspiration that shorter people and taller people may have different preferences with "up" and "down" position. The first experiment is to test the premise that taller people feel more comfortable looking down and shorter people feel more comfortable looking up. The following experiment is expected to be testing whether the association of good with up is weaker in tall people or stronger in tall people. Inspired by Casasanto and Chrysikou, in the third experiment, the artificial adjustment of people's bodily characteristics is considered as an important factor that could temporally change people's body specificity. The flexibility of the possible results in Experiment 1 and 2-whether manipulating participants to be tall or short can also reach the same results that heights moderate the overall association of good with up-is tested with the assistance of visual reality (VR) technique (Yee, Bailenson, \& Ducheneaut, 2009). We decide to include The VR machine can temporarily change the participants' height by having them experience being either tall or short.

Though using a new test bed to prove that height will affect people's judgment of positive and negative valence on different vertical vitual field, the present study aims to testify the body-specificity on valence judgment. The expected results will provide some evidence to the view that "good is up" metaphor is also influenced by individual's bodily experience, therefore give support to body-specificity hypothesis, which may give some hint about how human cognitive process is grounded.

\section{Methods}

\subsection{Experiment 1}

The pre-test task aims to indicate that taller people are more likely to look up and shorter people are more likely to look down. The experiment will verify the influences of people's height on their judgment of valence on vertical direction only if this premise was proved. In this task, participants should choose a line on the wall that they see most comfortable with.

\section{Participants}

36 US university students and staff participate in this study. They are divided into 2 groups: a taller group and a shorter group. 18 of them in the taller group are higher than 72.0 inches, which is much higher than the average height of American adult male; and 18 of them in the shorter group are shorter than 62.0 
inches, which is shorter than the average height of American adult female. In the United States, the average height for an adult male over age 20 is 69.2 inches (175.7 centimeters), and the average height for an adult female over age 20 is 63.7 inches (161.8 centimeters), according to the data from Centers for Disease Control and Prevention (CDC) (Fryar, et al., 2016). All participants are found physically and mentally fit and they do not have history of eye disease. Some of them have mild myopia with corrected visual acuity of 1.5 .

\section{Material and Procedure}

A white wall in an empty room with 10 feet tall serves as a material of this study. There are horizontal blue lines which are $85 \mathrm{~cm}$ long and $7 \mathrm{~mm}$ wide stuck to the wall from the bottom to ceiling. The space between the horizontal axes of two adjacent lines is $10 \mathrm{~cm}$ so that the lowest line is $0 \mathrm{~cm}$ tall and the highest line is $300 \mathrm{~cm}$ tall. There is a cross mark on the floor in front of the lines and $1.2 \mathrm{~m}$ from the wall. The participants are instructed to stand on the cross mark and use a laser pointer to point out which of those lines is the one they are most comfortable to look at their familiar visual angle. After the height-decision task, the experimenter measures the height of their eye level in another room.

\section{Predictions}

We are expected that the majority of the tall people (taller than 72 inches) choose the line lower than their eye levels, and majority of the shorter people (shorter than 62 inches) choose the line higher than their eye levels. If this data pattern show any significance, then it is confirmed that tall people are more likely to look up and short people are more likely to look down.

\subsection{Experiment 2}

The paper-and-pencil diagram task in Casasanto's (2009) study is used as a model of the present experiment. Based on the prediction that the pre-test has a significant effect, we assume that tall people prefer to look down, and short people like to look up. This experiment further verified the impact of people's height on the valence judgment of the vertical visual space.

\section{Participants}

The researchers selected 60 US university students as participants to the study, and participants are assigned to the valence judgment task. The participants are assigned to a taller group and a shorter group according to their height. 30 of participants in the taller group are higher than 72.0 inches, which is much higher than the average height of American adult male; and 30 of them in the shorter group are shorter than 62.0 inches, which is shorter than the average height of American adult female. All participants are physically and mentally healthy and do not have history of eye disease. Some of them have mild myopia with corrected visual acuity of 1.5 .

\section{Materials and Procedure}

Both the taller group and the shorter group accomplish the identical task. Participants are given pencil and paper with experimental instructions on one side 
of the page. Participants are first asked to read the instructions with a view to have a better understanding of what they were expected to do. The instructions inform the participants that later they will be given another piece of experiment paper with a cartoon figure on it about a child called Darryl planned to visit the zoo. There are two boxes on the paper; one is at the top of the paper and the other at the bottom. Darryl loves pandas (or tigers, dogs) and think they are good, but hates pandas (or lions, giraffes) and think they are bad (or vice versa). Both the animal groups and Darryl's preference in the certain animal group are counterbalanced. Five minutes later, the researchers send the experiment paper. Their task is to draw the animal Darryl loves into the box that represents good and draw the animal Darryl hates in the box that represents bad in a comfortable way.

The assignment of valence to the different groups balances across participants. Both the animal groups and Darryl's preference in the certain animal group are counterbalanced (Casasanto 2009).

\section{Predictions}

According to previous research by Casasanto (2009), there will be significant "up is good" effect in both taller and shorter group-there is a large proportion of participants related upper position with positive valence and lower with negative. If an individual's height has an interaction with the "up is good" effect, then in the taller group will less likely to draw the good animal in the upper box and shorter group are more likely to draw the good animal in the upper box.

\subsection{Experiment 3}

Based on the results in Experiment 2, Experiment 3 is an attempt to test the flexibility of the previous results, that is, whether manipulating participants to be taller or shorter can also give the same results that heights moderate the overall association of good with up-the proportion of individuals relating good with up in taller group is lower than the proportion in shorter group. The virtual reality technology which was adopted previously in Yee et al. (2009) study provides the possibility of manipulating the height of the participants. The data show some indication of the causal relationship between the individual's height and the moderation of the intrinsic pattern of "good is up". Experiment 2 still adopts to the identical Bob-go-to-the-zoo design in Casasanto's (2009) study.

\section{Participants}

72 US university students are hired to serve as participants of the experiment. They are randomly assigned to taller and shorter groups. All participants are physically and mentally healthy, with visual acuity of 1.5 and without a history of any eye disease.

\section{Material and Procedure}

Virtual reality technology is served as the approach of the height manipulation of this experiment. A virtual reality program that consists of four different scenes is designed to be the approach of manipulating participants' height. Each 
participant is asked to wear a pair of VR glasses and walk around in a big empty room. The experimenter advises the participants not to go too close to the walls. While wandering, participants see 4 different VR scenes (walking at a busy block, a nature park, a shopping mall, and a room with plenty of furnishings) and successively experience each for 10 minutes (random sequence). The taller group view the VR scenes from a tall people's perspective $(185 \mathrm{~cm}$ from the ground), and the shorter group observed the VR scenes from a short people's perspective (155 $\mathrm{cm}$ from the ground). Then participants are instructed to accomplish the same task in Experiment 2.

\section{Predictions}

If the height manipulation influences individuals' valence judgment and height have a causal relation with this interaction, it is assumed that after height manipulation, the proportion of individuals regarding up as a positive position in the taller group will be significantly lower than that in the shorter group.

\section{Discussion}

In Experiment 1, if most of the taller people choose the line lower than their eye levels, and most of the shorter people choose the line higher than their eye levels, we can conclude that taller people feel more comfortable to look downward and shorter people feel more comfortable to look upward. This is consistent with our assumption. The second and the third experiment verify the potential interaction between people's height and their tendency to associate "up" with "good" and "down" with "bad". If Experiment 2 and Experiment 3 expectantly have the same data patterns that the taller group have less association with up position with good animal and shorter group are more likely to associate up position with good animal, compared to the normal pattern showed in Casasanto's (2009) study, it is a strong evidence that height has impact on people's valence judgment on vertical visual space-the taller group associate more positive feelings with things in their lower visual field and more negative feelings with things in their higher visual field, whereas the shorter group associate more positive feelings with things in their upper visual field and more negative feelings with things in their lower visual field. The flexibility showed in Experiment 3 proves that the manipulation of people's height also influences the valence judgment on vertical visual space. If the difference between the present results and the data in previous research is statistically significant, then within the group of taller and shorter participants, the effect of interaction is significant, which supports the body-specificity hypothesis.

The results validate the body-specificity hypothesis in a new test bed beyond handedness. Consistent with the body-specificity hypothesis, people with different kinds of bodies interact with their surrounding environment in systematically different ways, as such create different forms of representation of the association between valence and space. Previous studies find about $90 \%$ of participants follows "Up is Good, Down is Bad" metaphor (Casasanto, 2009). However, 
if the results of the present study show the pattern as predicted, it will suggest people's height being a moderator of this effect. With the premise that taller people more comfortably look downward and shorter people do so upward, we assume perceptual and motor fluency plays a significant role in determining this preference, and the third experiment in a way indicate the causal relations between the height and this preference, since the height manipulation is former than the valence judgment task. Thus, we can interpret the moderation as a result of the different ways taller people and shorter people interact with their environment. Consistent with the body-specificity hypothesis, people with different kinds of bodies interact with their surrounding environment in systematically different ways, therefore create different forms of representation of the association between valence and space.

Further research may need to focus on ruling out the possibility of people imagining or expecting that they are taller or shorter instead of being manipulated in their real experience of height (Fuente et al., 2017). If the interaction is significant in the taller group when they are manipulated by virtual reality, while in the virtual manipulation situation, the interaction disappears or not is as significant as the manipulated situation, it may suggest whether the result in the third experiment is due to the actual bodily experience of the participants or their mere familiarity of different visual angle.

\section{Conclusion}

According to the aforementioned previous studies, we are inspired to reach out a method that could test the body-specificity without relying on handedness. In using heights as a test bed, the present proposal aims to verify body-specificity theory. If all of our assumptions in the three experiments proved to be true, then we could conclude that the valance judgment on vertical visual space is body-specific, which means, to be more specific, that taller people feel more comfortable to look downward and shorter people feel more comfortable to look upward, and this different preference among people with different heigh has an impact on their valence judgment on vertical visual space. In addition, the significance of the results would suggest that the method we proposed is promising in supporting body-specificity theory.

\section{Acknowledgements}

First and foremost, we would like to show our deepest gratitude to our supervisor, Prof. Casasanto, a respectable, responsible and resourceful scholar, who has provided us with much valuable guidance in every stage of the writing of this thesis. His keen and vigorous academic observation enlightens us not only in this thesis but also in our future study. We would like to extend our thanks to our TA Josephine for all her kindness and help. We would also like to thank one of our group-mates Li Yuan, who had together developed the thesis. Our sincere appreciation also goes to CIS scientific research for providing us chance to do scientific research with the help of world-class scholar and for other great coop- 
eration.

\section{Conflicts of Interest}

The authors declare no conflicts of interest regarding the publication of this paper.

\section{References}

Barsalou, L. W., Kyle Simmons, W., Barbey, A. K., \& Wilson, C. D. (2003). Grounding Conceptual Knowledge in Modality-Specific Systems. Trends in Cognitive Sciences, 7, 84-91. https://doi.org/10.1016/S1364-6613(02)00029-3

Casasanto, D. (2009). Embodiment of Abstract Concepts: Good and Bad in Right- and Left-Handers. Journal of Experimental Psychology: General, 138, 351-367. https://doi.org/10.1037/a0015854

Casasanto, D., \& Chrysikou, E. G. (2011). When Left Is "Right": Motor Fluency Shapes Abstract Concepts. Psychological Science, 22, 419-422. https://doi.org/10.1177/0956797611401755

Fryar, C. D., Gu, Q., Ogden, C. L., \& Flegal, K. M. (2016). Anthropometric Reference Data for Children and Adults: United States, 2011-2014. National Center for Health Statistics. Vital and Health Statistics 3, No. 39, 1-46.

Fuente, J., Casasanto, D., Martínez, C. J. I., \& Santiago, J. (2017). Motor Imagery Shapes Abstract Concepts. Cognitive Science, 41, 1350-1360. https://doi.org/10.1111/cogs.12406

Lakoff, G., \& Johnson, M. (1980). Metaphors We Live by. Chicago, IL: University of Chicago Press.

Yee, N., Bailenson, J. N., \& Ducheneaut, N. (2009). The Proteus Effect Implications of Transformed Digital Self-Representation on Online and Offline Behavior. Communication Research, 36, 285-312. https://doi.org/10.1177/0093650208330254 